\title{
Very High-Frequency Ultrasonography Signs in Bilateral Acute Choroidal Effusion Associated with Systemic Sulphonamide Medications: 2 Cases
}

\author{
Antonio Piñeiro Ces ${ }^{a}$ b, c Manuel Bande Rodriguez ${ }^{a, b}$ \\ María Rodriguez Blanco ${ }^{a}$ Lourdes Peña Madrid ${ }^{a}$ \\ María J. Lopez Valladares ${ }^{a}$ María J. Blanco Teijeiro ${ }^{a, b, c}$ \\ aUnidad de Retina Quirúrgica y Tumores Intraoculares del Adulto (URQTIA), Área Sanitaria \\ de Santiago de Compostela y Barbanza, Complejo Hospitalario Universitario de Santiago \\ de Compostela, Servizo Galego de Saúde (SERGAS), A Coruña, Spain; 'baboratorio de \\ Tumores Intraoculares del Adulto (IDIS), A Coruña, Spain; 'Departamento de Cirugía y \\ Especialidades Médico Quirúrgicas, Área de Oftalmología, Universidade de Santiago de \\ Compostela, A Coruña, Spain
}

\section{Keywords}

Acute angle closure · Choroidal effusion · Glaucoma · Imaging · Sulphonamide drugs

\begin{abstract}
Sulphonamides are commonly used in medicine for several purposes; however, they can lead to significant adverse effects, including idiosyncratic reactions and choroidal detachment corresponding to a forward rotation of the iris-lens diaphragm; this could also evolve into acute transient myopia with possible acute angle closure glaucoma. The risk of such reactions to sulphonamides is approximately $3 \%$. In our communication, we have reported on 2 cases involving patients who suffered choroidal detachments after starting sulphonamide treatments and who were diagnosed with the help of ultrasound biomicroscopy. Patient 1 was an 87-year-old male with bilateral pseudophakia who developed an acute change in vision in both eyes after he started taking chlorthalidone, a classic thiazide diuretic antihypertensive that is characterized by having a sulpha-based group. Patient 2 was a 42-year-old female who developed dramatic visual loss after beginning a new treatment (topiramate) for weight loss. We were able to successfully detect the choroidal detachments in these patients with ultrasound biomicroscopy, which aided us in quickly diagnosing the condition. Subsequently, the drugs were immediately discontinued, and appropriate treatment was administered resulting in the full recovery of both patients.
\end{abstract}




\section{Introduction}

Idiosyncratic drug reactions are adverse reactions that do not occur in most patients at any dose and do not involve the known pharmacological properties of the drug. Sulphonamide medications have 3 main indications: antidiabetic, diuretic, and antibiotic (however limited by important resistances), and some other ones. They have a number of well-known minor side effects. However, they can also lead to adverse effects, mainly allergic reactions, more or less severe. Ocular side effects are described including keratitis, ocular inflammation, and even possible permanent loss of vision. Moreover, idiosyncratic reactions have described resulting in choroidal detachment corresponding to an irido-ciliary-lenticular block. This phenomenon can evolve into acute transient myopia with possible acute angle closure (AAC) glaucoma as well as visual field alterations or maculopathies secondary to treatment with these drugs [1-3]. The risk of a reaction involving this mechanism to a sulphonamide is approximately $3 \%$ [4]. This report provides details of 2 cases presenting with an idiosyncratic reaction to different sulphonamides. The patients experienced choroidal detachments associated with forward rotation of the irido-ciliary-lenticular block to varying degrees; however, the exact mechanism remains controversial.

\section{Case Series}

This study followed the Declaration of Helsinki and received the territorial ethics committee approval (CEIC). Case 1 involved an 87-year-old male with bilateral pseudophakia (posterior chamber intraocular lens in the right eye and anterior chamber intraocular lens in the left eye) who developed an acute change in vision in both eyes. He was being treated with furosemide for arterial hypertension, and this treatment had been maintained without any issues for $>20$ years. On the day of the onset of the loss of visual acuity, his primary care physician recommended changing his hypertension medication to chlorthalidone, a classic thiazide diuretic antihypertensive that is characterized by a sulpha-based group. During the initial examination, his visual acuity was limited to hand movement in both eyes. The anterior segment and intraocular pressure (IOP) were normal. The corneas were clear without any evidence of oedema/decompensation (Fig. 1A). During the fundus examination, 2 massive temporal and nasal choroidal detachments were detected in both eyes (Fig. 1B). Ocular ultrasound (OU) examination revealed 2 bilateral, serous, and massive choroidal detachments with contact in the middle of the vitreous cavity (kiss sign) in both eyes (Fig. 1C). Very highfrequency ultrasonography (VHFU) revealed the association between the choroidal detachments and ciliary body oedema in the 4 quadrants of both eyes (Fig. 1D). The patient was diagnosed on the basis of clinical and ultrasonographic findings and treated with the oral corticosteroid prednisone (1 $\mathrm{mg} / \mathrm{kg}$ of weight) for 5 days. Chlorthalidone treatment was discontinued, and furosemide was restarted. Over an approximately 1-month period, the choroidal detachments flattened completely, and the patient recovered normal bilateral visual acuity.

Case 2 involved a 42-year-old female with no significant previous ocular history. She developed dramatic visual loss after beginning topiramate treatment for weight loss prescribed by a psychiatrist. Her previous treatment included olanzapine $10 \mathrm{mg}$, lorazepam $2 \mathrm{mg}$, and venlafaxine $150 \mathrm{mg}$ (retard) for several months. Subsequently, she began taking topiramate $(100 \mathrm{mg})$. One week later, she presented to the emergency department with visual acuity limited to hand movements in both eyes. The pinhole acuity was $6 / 12$ in both eyes.

Biomicroscopy revealed corneal oedema, conjunctival chemosis, a narrow anterior chamber, and angular closure confirmed by gonioscopy. The pupils exhibited light-reactive

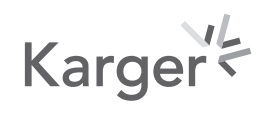




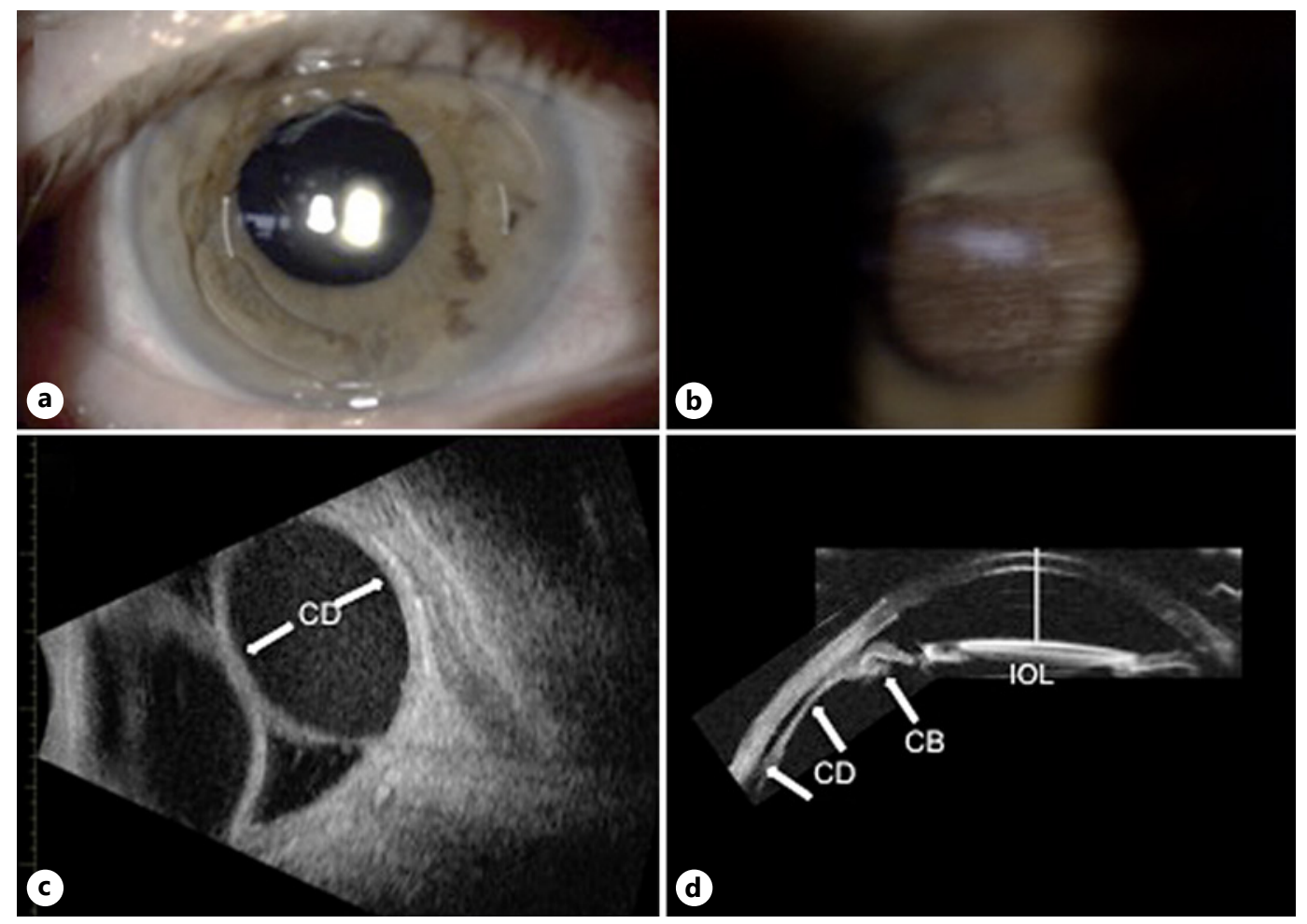

Fig. 1. a Image of the anterior segment of the left eye. Anterior chamber IOL can be detected. Loss of fundus reflex was easily detectable in both eyes. $\mathbf{b}$ Image of the anterior segment of the left eye showing the choroidal detachment in the middle of the pupil. c Ocular ultrasonography performed for diagnosis in the same left eye. The longitudinal projection showed a complete temporal and nasal area of CD. The "kiss sign" was present. d Reconstruction of VHFU images of the left eye of patient 1 one day after discontinuing the treatment with chlorthalidone. The anterior chamber IOL was detectable. No diminution was found in the central axial depth (the distance from the apex epithelial cornea to the anterior surface of the intraocular lens was $3.5 \mathrm{~mm}$; arrow). Peripheral CDs and the CB were diagnosed. CD, choroidal detachment; IOL, intraocular lens; $\mathrm{CB}$, ciliary body; VHFU, very high-frequency ultrasonography.

mydriasis. The IOP was $55 \mathrm{~mm} \mathrm{Hg}$ in both eyes. Therefore, a diagnosis of bilateral AAC was made (Fig. 2A-D). Topical treatment with pilocarpine $2 \%$, timolol $0.5 \%$, and brimonidine $0.2 \%$ as well as 1 oral acetazolamide tablet was administered. After $2 \mathrm{~h}$, the IOP was 30 and $32 \mathrm{~mm} \mathrm{Hg}$, respectively. Subsequently, $24 \mathrm{~h}$ after the first visit, while her visual acuity remained unchanged, the patient felt better, the corneal oedema had disappeared, and the IOP was 28 and $30 \mathrm{~mm} \mathrm{Hg}$, respectively. OU and VHFU were performed and revealed an angular closure, a narrow anterior chamber, and a uveal effusion that pushed the irido-ciliarylenticular block in both eyes. The images obtained with VHFU were not compatible with manifestations of a plateau iris, since although the ciliary body was rotated forward, the main symptom was the complete narrowing of the anterior chamber caused by a spherical change in the lens.

Consequently, patient 2 was diagnosed with secondary angle closure associated with a ciliochoroidal detachment that was suspected to result from topiramate use. Pilocarpine was discontinued at this time, but the IOP continued to be managed with timolol and brimonidine. In agreement with the prescribing doctor, topiramate was discontinued. Five days after discontinuation of topiramate, the patient's condition continued to evolve adequately, and her best-corrected visual acuity was $6 / 12$ in both eyes, which improved to $6 / 6$ with a -2 


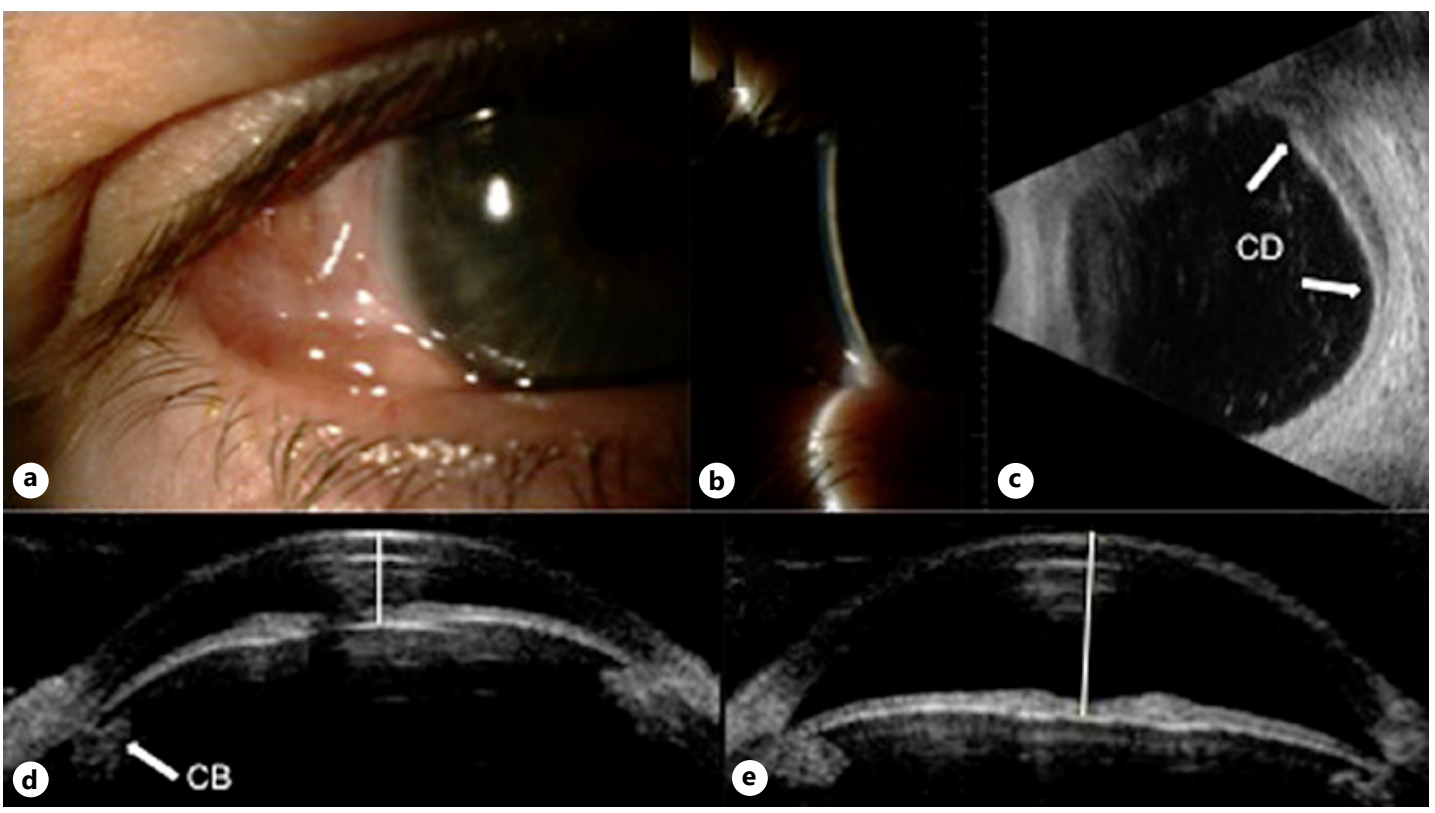

Fig. 2. a Corneal oedema and conjunctival chemosis was detected in patient 2. b Slit lamp photograph disclosing narrow anterior chamber and angular closure. c Image of the CD obtained by ocular ultrasonography in the temporal meridian of the RE. $\mathbf{d}$ VHFU on the day of acute angle glaucoma. The anterior displacement of the iris-lens diaphragm is very important. The central axial depth was narrowing the anterior chamber (the distance from the apex of the epithelial cornea to the anterior surface lens was $1.8 \mathrm{~mm}$; arrow). The signs indicating the anterior rotation of the $\mathrm{CB}$, and the serous CDs in the 4 quadrants were clearly detected. e VHFU of the same regions performed 1 month after. The images are axial and horizontal. Although height figures are added, these measurements are not exactly comparable as the cuts are not exactly the same. CD, choroidal detachment; CB, ciliary body; VHFU, very high-frequency ultrasonography.

spherical graduation. The IOP was 10 and $12 \mathrm{~mm} \mathrm{Hg}$, respectively. VHFU was performed again, and total disappearance of the uveal effusion was evident; in addition, the central axial depth and opening of the angle were normal (Fig. 2E). Both the optical discs were normal. The patient exhibited normal visual function 1 month later.

\section{Discussion}

The situations of acute and bilateral choroidal effusion in relation to idiosyncratic reactions to different drugs are well known. The clinic includes bilateral involvement with blurring of vision acuity, which generally occurs over minutes to hours, nausea or vomiting, red eyes, and headache. An examination might reveal conjunctival injection, corneal oedema, anterior chamber inflammation, and a flat or shallow anterior chamber. Bilateral AAC glaucoma is observed in some patients. These patients exhibit very specific clinical characteristics, and the use of OU examinations, especially VHFU, is important to make a diagnosis.

The principal therapy in such cases is prompt discontinuation of the drug involved. Generally, topical miotics and peripheral iridectomy are not helpful. If the IOP remains uncontrolled, additional therapies, such as topical IOP-lowering medications, high-dose steroids, and trabeculectomy, may need to be considered [1-3].

The exact aetiopathogenic mechanism underlying these cases is not known, but it is believed that these symptoms could be an idiosyncratic reaction to the drug molecules. One 
potential critical aspect is the synthesis of prostaglandin E2, which particularly stimulates vasodilatation and can increase vascular permeability in the anterior uvea [1]. Thus, bilateral AAC related to glaucoma and transient myopia (anterior displacement of the irido-ciliarylenticular block) has been described as a progression of the choroidal effusion. Several drugs have been associated with these conditions: adrenergic, anticholinergic, antihistaminic, and cholinergic agents, antidepressants [5], anxiolytics, anticoagulants, and sulphabased drugs (acetazolamide [6], hydrochlorothiazide [2], cotrimoxazole, sulphamethoxazole, sulphasalazine, indapamide [7], and topiramate [8]).

In both clinical cases presented in this article, the ocular reactions to the sulpha-based drugs were very similar. Both patients started new clinical treatments, despite very different clinical indications. Patient 1 changed his anti-hypertension treatment to chlorthalidone, a classic diuretic drug that acts by lowering peripheral vascular resistance and inhibiting the ability of the kidneys to retain water [2]. This patient also received a single dose of acetazolamide (250 mg); however, this dose was not considered to worsen the progression of the AAC glaucoma. Patient 2 began treatment with topiramate as a weight loss drug ("off-label" treatment). Furthermore, for years, this patient was treated with another psychotropic drug, venlafaxine, which is a selective serotonin and norepinephrine reuptake inhibitor antidepressant and has also been associated with choroidal effusions and AAC in some cases. In this case, it appears that the choroidal effusion was attributable to the addition of topiramate and not the venlafaxine treatment [5] that was being administered for several years without problems.

Contrarily, the presence of AAC glaucoma may be related to the anatomy of the iridocorneal angle: thus, pseudophakia patients (case 1) are at a lower risk, and phakic patients with a narrow angle (case 2) are at a high risk of developing AAC. A very similar case to that of patient 1 was described in relation to indapamide [7]. The architecture of each eye structure and its reaction to these drugs were effectively revealed using ultrasound, and the overall findings were shown using VHFU. Uveal effusion syndrome and, in some cases, ciliary body oedema can cause anterior displacement of the irido-ciliary-lenticular block leading to myopia, narrowing of the anterior chamber, and AAC crisis. VHFU can facilitate our understanding of the physiopathological processes involved in this rare syndrome. In these patients, discontinuation of the treatment is the fundamental step for blocking the pathogenesis. As demonstrated in several studies, VHFU is much more important than optical coherence tomography for anterior segment examinations, especially to monitor the degree of choroid detachment and the anterior rotation of the ciliary body $[9,10]$.

Potentially, the most effective step in cases involving the occurrence of choroidal and ciliary body detachment and AAC glaucoma is to withdraw the drug responsible. Patient 1 was treated with oral corticosteroids for reabsorption of choroidal effusion; however, this was not very effective. Further, iridectomy and miotics are not effective in treating this type of closed glaucoma, as they do not induce pupillary block [1-3]. Cycloplegics agents, not used in these cases, are effective in improving cilichoroidal effusion and reducing IOP in this type of angle closure glaucoma cases, since they cause retraction of the ciliary processes and induce an anatomic deeper anterior chamber $[2,3]$.

\section{Statement of Ethics}

The authors of this work declare that this experiment complied with the guidelines for human studies and was in accordance with the World Medical Association Declaration of Helsinki. Written informed consent was obtained from the patients for publication of this case report and any accompanying images. Any sort of information that might reveal the

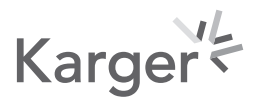


patient's own identity has been completely avoided. This study protocol was reviewed and approved by the Galicia Research Ethics Committee in January 2009, affiliated with Servicio Galego de Saude.

\section{Conflict of Interest Statement}

The authors have no conflicts of interest to declare.

\section{Funding Sources}

This study was funded by Instituto de la Salud Carlos III/PI16/00143/Cofound by FEDER.

\section{Author Contributions}

The authors confirm sole responsibility for the following: study conception and design, data collection, analysis and interpretation of results, and manuscript preparation. Dr. Antonio Piñeiro-Ces contributed to design, data collection, analysis and interpretation of results, and manuscript preparation. Dr. Manuel Bande-Rodriguez contributed to data collection. Dr. María Rodriguez-Blanco contributed to data collection. Dr. Lourdes PeñaMadrid contributed to data collection. Dr. María J. Lopez-Valladares contributed to data collection. Dr. María J. Blanco-Teijeiro contributed to data collection.

\section{Data Availability Statement}

The authors confirm that the data supporting the findings of this study are available within the article.

\section{References}

1 Krieg PH, Schipper I. Drug-induced ciliary body oedema: a new theory. Eye. 1996;10(Pt 1):121-6.

2 Söylev MF, Green RL, Feldon SE. Choroidal effusion as a mechanism for transient myopia induced by hydrochlorothiazide and triamterene. Am J Ophthalmol. 1995;120:395-7.

3 Lachkar Y, Bouassida W. Drug-induced acute angle closure glaucoma. Curr Opin Ophthalmol. 2007;18:129-33.

4 Panday VA, Rhee DJ. Review of sulfonamide-induced acute myopia and acute bilateral angle-closure glaucoma. Compr Ophthalmol Update. 2007;8:271-6.

5 De Guzman MH, Thiagalingam S, Ong PY, Goldberg I. Bilateral acute angle closure caused by supraciliary effusions associated with venlafaxine intake. Med J Aust. 2005;182:121-3.

6 Malagola R, Arrico L, Giannotti R, Potavina N. Acetazolamide induced cilio-choroidal effusion after cataract surgery: unusual posterior involvement. Drug Des Devel Ther. 2013;7:33-6.

7 Phylactou M, Matarazzo F, Jones E. Indapamide-induced bilateral choroidal effusion in pseudophakic patient. BMJ Case Reports. 2018;2018:bcr2018225920.

8 Asensio-Sánchez VM, Torreblanca-Agüera B, Martínez-Calvo S, Calvo MJ, Rodríguez R. (Severe ocular side effects with topamax). Arch Soc Esp Oftalmol. 2006;81:345-8.

9 Waheeb S, Feldman F, Velos P, Pavlin CJ. Ultrasound biomicroscopic analysis of drug-induced bilateral angleclosure glaucoma associated with supraciliary choroidal effusion. Can J Ophthalmol. 2003;38:299-302.

10 Mitra A, Ramakrishnan R, Kader MA. Anterior segment optical coherence tomography documentation of a case of topiramate induced acute angle closure. Indian J Ophthalmol. 2014;62:619-22. 